\title{
La cinugía de revascularización miocártica se asocia a una menor incidencia de eventos cardiovasculares que la angioplastía transluminal coronaria con colocación de stents liberadores de everolimus
}

\section{Coronary artery bypass grafting was associated with lower rate of adverse cardiovascular events than percutaneous}

coronary intervention with everolimus-eluting stents implantation

Park SJ, y col. NEJM 2015;372:1204-12.

\section{Objetivos}

Comparar en pacientes con enfermedad coronaria de múltiples vasos (ECMV), los resultados a dos años del tratamiento con angioplastia transluminal coronaria (ATC) con colocación de stents liberadores de everolimus versus cirugía de revascularización miocárdica (CRM).

\section{Diseño}

El estudio BEST es un ensayo clínico aleatorizado multicéntrico (27 centros del este asiático con aleatorización realizada por centro), prospectivo, abierto y de no inferioridad. Fueron incluidos mayores de 18 años con ECMV confirmada por cinecoronariogrfía (CCG) con lesiones mayores al $70 \%$ en al menos dos arterias coronarias. Fueron excluidos los pacientes con enfermedad significativa del tronco de la coronaria izquierda. Las CCG fueron evaluadas por cardiólogos y cirujanos.

\section{Intervención y medición de resultados principales}

A los pacientes elegidos, se les realizó ATC con colocación de stents liberadores de everolimus o CRM, mayormente con la utilización de la arteria mamaria interna (AMI) a la descendente anterior (DA). El resultado primario fue un evento combinado de muerte, infarto de miocardio (IAM) y revascularización del vaso responsable; y los secundarios, un combinado de muerte, IAM, accidente cerebrovascular (ACV) y revascularización repetida. Los puntos finales secundarios adicionales fueron la trombosis del stent, el sangrado mayor o fatal y los componentes individuales de los resultados combinados. El análisis fue por intención de tratar.

\section{Resultados principales}

De los 1.776 pacientes evaluados, fueron reclutados 880 entre 2008 y 2013; 438 asignados a ATC y 442 a CRM. De los asig- nados a ATC y CRM, 413 y 382 pacientes recibieron el tratamiento asignado originalmente, respectivamente. $\mathrm{EI}$ comité de monitoreo de datos y seguridad recomendó suspender el reclutamiento por bajo flujo de pacientes al ensayo clínico.

El resultado primarios a los dos años de seguimiento ocurrió en $11 \%$ de los asignados a ATC y en $7,9 \%$ de los asignados a CRM (reducción de riesgo absoluto [RRA] 3,1; IC 95\%-0,8 a $6,9 ; p=0,32)$.

En el seguimiento a largo plazo -media de 4,6 años y con pérdida de tres pacientes- los puntos finales primarios fueron $15,3 \%$ para ATC vs $10,6 \%$ para CRM (HR 1,47; 1,01 a 2,13; $\mathrm{p}=0,04)$ y los secundarios fueron $19,9 \%$ para ATC y $13,3 \%$ para CRM $(p=0,01)$. Se observó una diferencia mayor en la incidencia de eventos primarios en quienes tenían diabetes (19,2\% para ATC y $9,1 \%$ para CRM, HR 2,$24 ; 1,25$ a 4,00$)$. Analizando los eventos individuales: la tasa de ACV no fue significativamente diferente entre el grupo asignado a ATC y CRM (2,5 y $2,9 \%$ respectivamente; $p=0.62)$. La incidencia de IAM espontáneo fue de $4,3 \%$ para ATC y de $1,6 \%$ para CRM $(p=0,02)$ y la de revascularización repetida, $11 \%$ para ATC vs $5,4 \%$ para CRM $(p=0,003)$. La tasa de sangrado mayor fue $6,8 \%$ en el grupo ATC y $29,9 \%$ en el grupo CRM $(p<0,001)$, sin diferencias en la tasa de sangrado fatal. La tasa de trombosis de los stents fue de $1,6 \%$.

\section{Conclusión}

En los pacientes con ECMV, la ATC con la colocación de stents de segunda generación liberadores de droga (Everolimus), se asoció a una tasa de ECV mayores que la CRM.

Fuente de financiamiento: Cardiovascular Research Foundation. Abbott Vascular y el Ministerio de Salud de Corea del Sur.

\section{Comentario}

Actualmente, debido a los avances técnicos en los stents, gran cantidad de los pacientes con ECMV se están tratando con ATC en lugar de CRM a pesar de que las guías actuales sugieren a esta última como tratamiento de mayor eficacia ${ }^{1,4,5}$. De acuerdo a los resultados del SYNTAX, el más significativo de los ensayos clínicos aleatorizados sobre el tema, la CRM debe permanecer como el estándar de tratamiento para la ECMV ya que sus resultados fueron superiores a los de ATC con stents liberadores de drogas de primera (Paclitaxel) y segunda generación (Everolimus) ${ }^{2,3}$.

El estudio que hemos resumido tuvo insuficiente poder para detectar el efecto diferencial de tratamiento entre grupos para variables individuales como muerte o IAM, ya que fue originalmente diseñado para evaluar un punto final combinado. Además, el poder estadístico fue menor ya que el estudio terminó tempranamente (fueron reclutados 880 pacientes en lugar de los 1776 estadísticamente necesarios). Finalmente el haber incluido sólo pacientes asiáticos puede afectar la generalización de los hallazgos.

\section{Conclusiones del comentador}

Las guías actuales brindan excelentes herramientas para tomar decisiones respecto de las a estrategias de revascularización en ECMV. La superioridad de la CRM frente a la ATC está respaldada por múltiples ensayos y registros internacionales, quedando reservada la ATC sólo para pacientes con enfermedad leve, sabiendo que serán necesarias más reintervenciones- y en quienes la CRM se asocia con alto riesgo operatorio debido a sus comorbilidades. La CRM debe permanecer como el estándar de tratamiento para la ECMV, especialmente en pacientes con diabetes y enfermedad intermedia y compleja.

\section{José Gaspar Chas [ Servicio Cirugía Cardiovascular del Hospital Italiano de Buenos Aires. jose.chas@ hospitalitaliano.org.ar]}

Chas J. La cirugía de revascularización miocárdica se asocia a una menor incidencia de eventos cardiovasculares que la angioplastía transluminal coronaria con colocación de stents liberadores de everolimus. Evid Act Pract Ambul. 2016;19(3):80. Park SJ, y col. Trial of Everolimus-Eluting Stents or Bypass Surgery for Coronary Disease; N Engl J Med 2015; 372:1204-12. PMID: 25774645

\section{Referencia}

1.Serruys $\mathrm{P}$ y col. Percutaneous coronary intervention versus coronary-artery bypass grafting for severe coronary artery disease. N Engl J Med $2009 ; 360: 961-72$. 2. Mohr F y col. Coronary artery bypass graft surgery versus percutaneous coronary intervention in patients with three-vessel disease and left main coronary disease: 5 -year follow-up of the randomized, clinical SYNTAX trial. Lancet 2013;381: 629-38.

3. $\mathrm{H}$ y col. 5-year results of randomized comparison of XIENCE V everolimus-eluting and TAXUS paclitaxel-eluting stents: final results from the SPIRIT III trial (clinical evaluation of the XIENCE V everolimus eluting coronary stent system in the treatment of patients with de novo native coronary artery lesions). JACC Cardiovasc Interv 2013;6: 1263-6.

4. Manesh R y col. Appropiate Use Criteria for Coronary Revascularization Focused Update. JACC 2012; 59; 9: 857-881.

5. Windecker S y col. ESC/EACTS Guidelines on myocardial revascularization: The Task Force on Myocardial Revascularization of The European Society of Cardiology and the European Association for Cardiology-Thoracic Surgery, developed with the special contribution of the European Asociation of Percutaneous Cardiovascular Interventions. Eur Heart J 2014; 35:2541-619. 